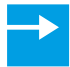

Wie viel verdienen Ergotherapeuten in den USA, in welchen Tätigkeitsfeldern arbeiten sie und wie entwickelt sich der Beruf dort? Dazu gibt der US-amerikanische Berufsverband American Occupational Therapy Association (AOTA) regelmäßig Auskunft - aktuell anhand einer Befragung von über 15.000 Ergotherapeuten [1].

Darüber hinaus veröffentlicht die AOTA evidenzbasierte Praxisleitlinien. Zum Beispiel für die Behandlung von Menschen mit schweren psychischen Erkrankungen [2] oder mit einer Autismus-Spektrum-Störung [3]. Damit möchte der Berufsverband Ergotherapeuten dabei unterstützen, vor allem jene Behandlungsverfahren zu nutzen, die nachweislich wirksam sind. Zudem erfahren Kostenträger im Gesundheitssystem auf diese Weise, wann ein Beitrag der Ergotherapie zur Behandlung gerechtfertigt ist.

\section{Ergotherapie-Assistenten und Ergothera-} peuten $\rightarrow$ Die Professionalisierung der Ergotherapie hat in den Vereinigten Staaten zwei Berufe hervorgebracht [4]: Certified Occupational Therapy Asisstants (COTA) und Occupational Therapists, Registered (OTR) [5].

Ergotherapie-Assistenten benötigen mindestens den sogenannten Associate‘s Degree, den sie nach einem zweijährigen Studium an einer vom Accreditation Council for Occupatio- 


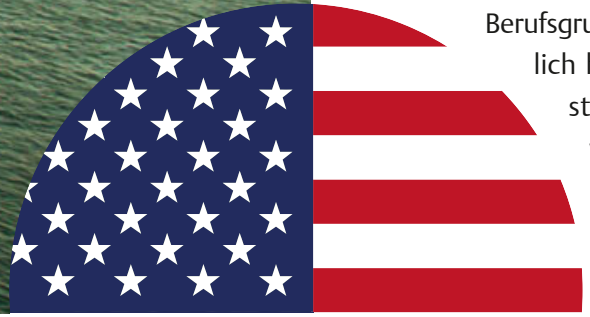
nal Therapy Education (ACOTE) anerkannten Hochschule erwerben [6]. Nach bestandener Prüfung dür-

fen sie die Berufsbezeichnung COTA führen.

Damit sind sie meist einem Ergotherapeuten unterstellt, der zum Beispiel die Diagnostik im Behandlungsprozess übernimmt. Mit Praxiserfahrung und regelmäßiger Supervision können Ergotherapie-Assistenten Behandlungen selbstständig durchführen.

Ergotherapeuten hingegen müssen mindestens ein Masterprogramm an einer akkreditierten Universität absolvieren, um zu praktizieren [7]. Für die Zulassung zu einem solchen Graduiertenstudium benötigt man einen Bachelorgrad mit naturwissenschaftlichem Fächerkanon. Der Masterstudiengang dauert dann in der Regel fünf Jahre. Eine weitere Voraussetzung sowohl für Master- als auch für Promotionsabschlüsse ist ein klinisches Praktikum in einem ergotherapeutischen Behandlungsfeld von mindestens 24 Wochen. ERGOTHERAPIE, S. 42):
Um den Titel OTR zu führen, benötigen Ergotherapeuten zur Berufsausübung eine bundesstaatliche Lizenz. Voraussetzung hierfür ist das Bestehen einer zentralen Aufnahmeprüfung nach Abschluss eines mindestens vierjährigen Studiums an einer akkreditierten Universität.

In manchen Bundesstaaten wird für die Berufsausübung der Doktorgrad Occupational Therapy Doctor (OTD) verlangt [6].

Steigender Fachkräftebedarf $\rightarrow$ Im Jahr 2017 waren mehr als 220.000 Ergotherapeuten und 64.000 Ergotherapie-Assistenten in den Vereinigten Staaten registriert [7]. Davon arbeiteten die meisten in den bevölkerungsreichen Bundesstaaten Kalifornien, Florida, Illinois, New York, Pennsylvania und Texas. Die Zahl der Ergotherapeuten wird bis 2024 voraussichtlich um 27 Prozent steigen [7]. Mit mehr als 30.000 neuen Stellen hat diese

lich hohe Wachstumsrate. Der steigende Fachkräftebedarf wird durch die Zunahme von Muskel- und Skelettsowie demenziellen Erkrankungen der Babyboomer-Generation erklärt, die das US-amerikanische Gesundheitssystem vor Herausforde-

$\rightarrow$ Zu-Hause-Behandlung in Form von mobiler Ergotherapie (Home Health): z. B. Gesundheitsfürsorge im häuslichen Lebensumfeld für Menschen mit Diabetes oder Schlaganfall

$\rightarrow$ Krankenhaus (Hospital): Ergotherapie der stationären Versorgung in somatischen Krankenhäusern, Rehabilitationsabteilungen oder im Hospiz

$\rightarrow$ stationäre Langzeitpflege (Long-Term Care/ Skilled Nursing Facility): z. B. Versorgung in subakuten Pflegeeinrichtungen für Erwachsene mit Schädel-Hirn-Trauma oder Demenz

$\rightarrow$ Psychiatrie (Mental Health): z. B. psychiatrisches Fachkrankenhaus, Tagesklinik oder andere gemeindepsychiatrische Träger

$\rightarrow$ Schulen (Schools): Ergotherapie an öffentlichen und privaten Schulen, z. B. für Jugendliche im Übergang zu Ausbildung und Beschäftigung

$\rightarrow$ Sonstige (Other): z. B. Leistungen der beruflichen Rehabilitation wie Supported Employment für schwer psychisch Erkrankte oder Fahrtraining für ältere Menschen

Ergotherapie gehört zum amerikanischen Schulsystem $\rightarrow$ Ein US-amerikanisches Bundesgesetz garantiert die therapeutische Versorgung für alle Schüler mit Behinderungen [4, 8]. Aus diesem Grund ist die Ergotherapie ein integraler Bestandteil des amerikanischen Schulsystems.

2014 waren 20 Prozent der Ergotherapeuten und 15 Prozent der Ergotherapie-Assistenten an Schulen angestellt. Dort arbeiten sie gemein-

\section{5} tätigkeitsfeldern erbracht ( $\odot$ ARBEITSFELDER

$\rightarrow$ Hochschultätigkeit (Academia): Forschung und Lehre an Universitäten

$\rightarrow$ gemeindenahe Ergotherapie (Community): z. B. für Erwachsene mit Sehschwäche oder ältere Menschen in der Gemeinde

$\rightarrow$ Frühintervention (Early Intervention): ergotherapeutische Behandlung in der frühen Kindheit von der Geburt bis 3 Jahre. Solche Programme beinhalten u. a. die Abklärung, Evaluation und Behandlung von motorischen, kognitiven, sozioemotionalen und adaptiven Entwicklungsstörungen der Kinder sowie die Beratung von Angehörigen.

$\rightarrow$ ambulante Versorgung (Freestanding Outpatient): z. B. Ergotherapie aus unabhängigen Kliniken privater Träger oder Praxen

\section{Mit mehr als 30.000 neuen Stellen hat die Ergotherapie eine überdurchschnittlich hohe Wachstumsrate.}

sam mit Lehrern in einem Team und unterstützen Kinder und Jugendliche in deren Rolle als Schüler [10]. Zum Beispiel mit außerunterrichtlichen Angeboten zur gesunden Lebensgestaltung, Beratung von Eltern zu Erziehungsfragen oder Sprach- und Bewegungsförderung. Ein Schwerpunkt liegt auf dem besonderen Förderbedarf bei Legasthenie oder Dyskalkulie. 


\section{Arbeitsfelder Ergotherapie}

In den USA arbeiten die meisten Ergotherapeuten im Krankenhaus (27\%).

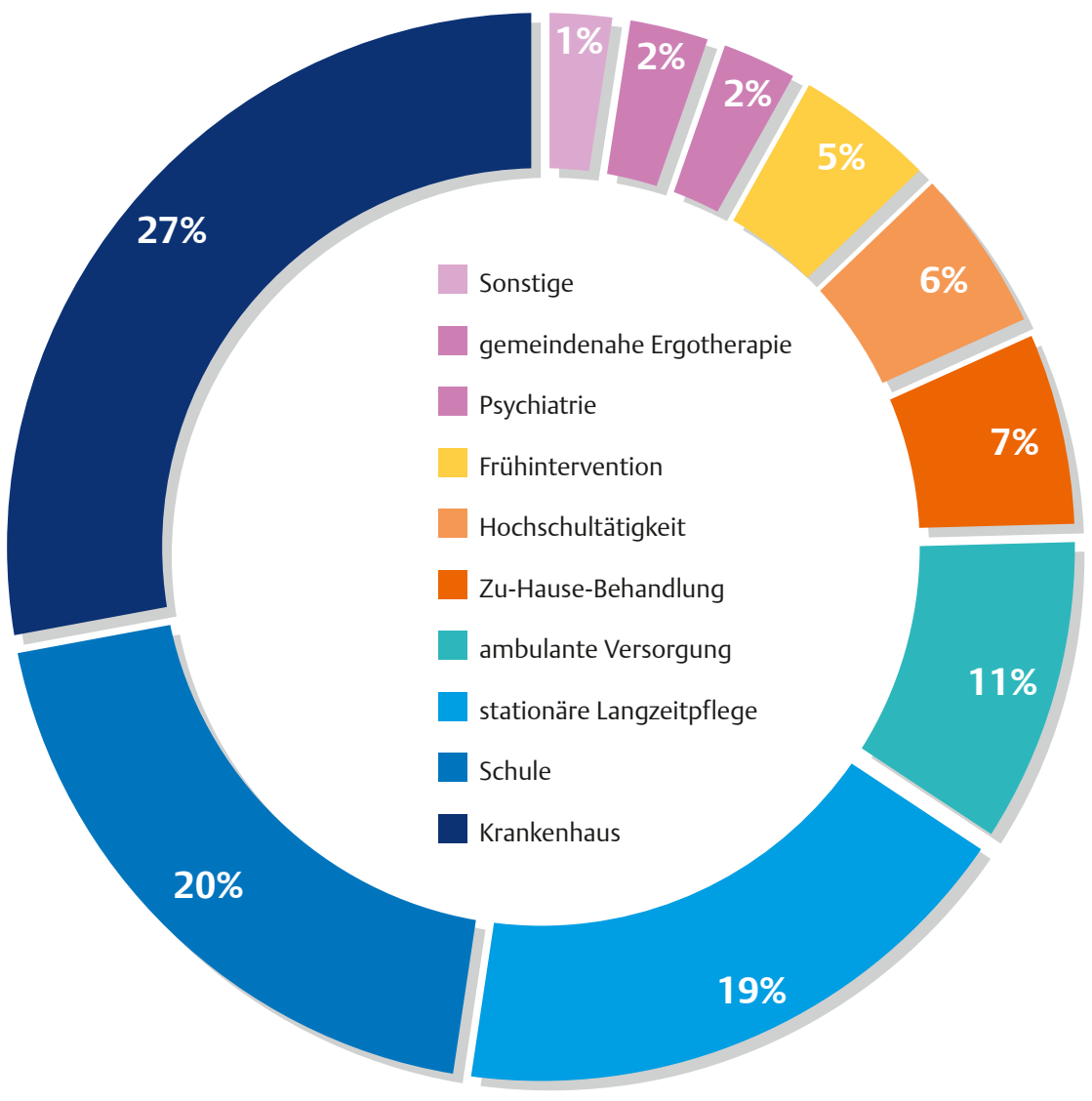

Quelle: American Occupational Therapy Association. 2015 AOTA Salary and Workforce Survey. Bethesda, MD: AOTA Press; 2015

\section{Arbeitsfelder mit den höchsten Gehältern}

Ergotherapeuten in Forschung und Lehre verdienen am meisten.

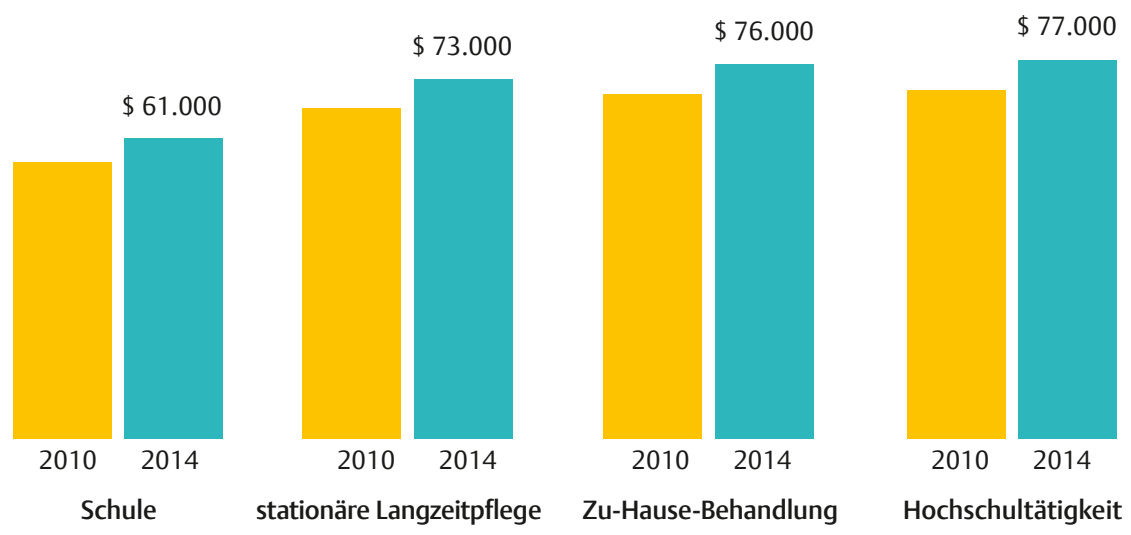

Quelle: American Occupational Therapy Association. 2015 AOTA Salary and Workforce Survey. Bethesda, MD: AOTA Press; 2015

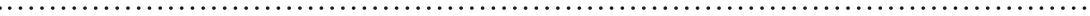

Dabei helfen Ergotherapeuten beim Lösen von Aufgaben im Unterricht, um die Lernperformanz zu verbessern. Zudem überprüfen sie mit standardisierten Tests die berufliche Eignung von Jugendlichen im Übergang von der Schule in eine Beschäftigung.

\section{Weniger Ergotherapeuten im Arbeitsfeld} Psychiatrie $\rightarrow$ In den USA gibt es im Gegensatz zu Deutschland keine versicherungsrechtliche Gleichstellung zwischen der Übernahme von Behandlungskosten bei somatischen und psychischen Erkrankungen [8]. Im Survey wird das Arbeitsfeld Psychiatrie deshalb extra aufgeführt und von der Ergotherapie in einem somatischen Krankenhaus abgegrenzt [1].

\section{5}

\section{Auch in den USA ist die Ergotherapie mit einem Anteil von knapp $91 \%$ weiblichen Berufsangehörigen ein Frauenberuf.}

Betrachtet man die Berufsentwicklung der vergangenen Jahre, so waren 2014 lediglich $2 \%$ der Ergotherapeuten in der Psychiatrie und Psychosomatik beschäftigt. Hingegen waren es im Jahr 2000 noch über 5\%. Zum Vergleich: In Deutschland arbeiten 14\% der vom DVE befragten Angestellten im Bereich Psychiatrie [11].

Allerdings lässt sich die psychiatrische Ergotherapie in den USA nicht immer klar abgrenzen. Berufliche Rehabilitationsleistungen wie JobCoaching nach dem Ansatz Supported Employment werden zum Beispiel dem Tätigkeitsfeld "Sonstige“ zugeordnet (॰ ARBEITSFELDER ERGOTHERAPIE). Dabei unterstützen Ergotherapeuten psychisch Erkrankte direkt und zeitlich unbefristet am Arbeitsplatz in der Gemeinde. Außerdem arbeiten Ergotherapeuten in Clubhouse-Einrichtungen, die zur "gemeindenahen Ergotherapie“ zählen. Im Clubhouse treffen sich - ähnlich wie in deutschen sozialpsychiatrischen Zentren - Klienten zu sozialen Aktivitäten und Arbeit, um sich auf den Übergang zum ersten Arbeitsmarkt vorzubereiten [12].

Der Rückgang der Beschäftigten innerhalb der psychiatrischen Ergotherapie wird gesund- 
heitspolitisch und wirtschaftlich begründet [13]. So hat man in der Vergangenheit versäumt, den Beitrag der Ergotherapie zur Behandlung von psychisch erkrankten Menschen für Gesetzgeber und Kostenträger mit gut kontrollierten Studien abzusichern. Zudem wechselten viele Therapeuten nach einer Gesetzesänderung in den 1970er Jahren von ihrem bisherigen Tätigkeitsfeld an Schulen. Ein weiterer Grund ist, dass viele Absolventen nach ihrer Universitätsausbildung durch Darlehen belastet sind und eine Tätigkeit im Arbeitsfeld Psychiatrie für sie nicht lukrativ ist [13]. Heute steuert der amerikanische Berufsverband diesem Trend mit Praxisleitlinien und international erfolgreichen Therapieansätzen entgegen.

\section{Arbeitsfeld „Langzeit-}

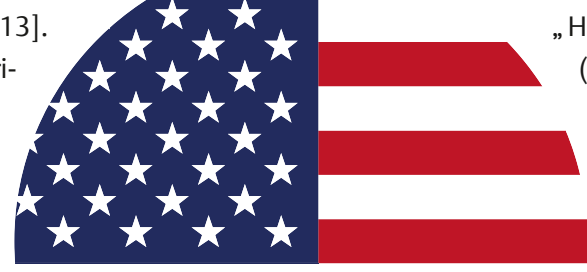
pflege“ wächst $\rightarrow$ Die

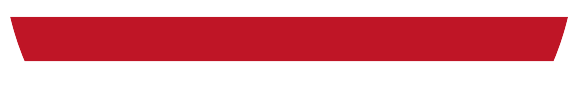

Mehrheit der Ergotherapeuten arbeitet in somatischen Krankenhäusern (27\%), an Schulen (20\%) und in der stationären Langzeitpflege (19\%) (○ ARBEITSFELDER ERGOTHERAPIE). Dabei unterscheidet sich die Berufsgruppe der Ergotherapeuten von der der Ergotherapie-Assistenten erheblich: So sind mehr als 55\% der Assistenten in Langzeiteinrichtungen tätig. Hingegen liegt der Anteil von Ergotherapeuten mit einem

\section{y5}

\section{Die Zahl der Ergothera- peuten wird in den USA bis 2024 voraussichtlich um 27 Prozent steigen.}

höheren Abschluss in diesem Arbeitsfeld bei weniger als $20 \%$. Insgesamt wächst dieser Sektor jedoch für beide Berufsgruppen am schnellsten.

Mehr Masterabschlüsse und steigende Gehälter $\rightarrow$ Seit Einführung des Masterabschlusses als Voraussetzung für den Berufseinstieg im Jahr 2007 hat sich die Anzahl der
Ergotherapeuten mit Master von 31,9\% im Jahr 2006 auf $60 \%$ im Jahr 2014 nahezu verdoppelt.

Im Vergleich zur letzten Erhebung sind auch die Gehälter beider Berufsgruppen gestiegen: um 8,2\% bei den Ergotherapeuten und um $9,1 \%$ bei den Ergotherapie-Assistenten. Als Faustformel gilt: Je höher der akademische Abschluss, desto höher das Jahresgehalt. So variiert die Vergütung eines Ergotherapeuten mit Master zwischen 75.000 und 88.000 \$1]. Die höchsten Durchschnittsgehälter werden Ergotherapeuten in der "Hochschultätigkeit“ $(77.000 \$)$, in der „ZuHause-Behandlung “ $(76.000 \$)$ und in der „stationären Langzeitpflege“ (73.000\$) gezahlt. Schulergotherapeuten verdienen mit einem Jahresgehalt von $61.000 \$$ am wenigsten ( $\odot$ ARBEITSFELDER MIT DEN HÖCHSTEN GEHÄLTERN) [1].

Das Durchschnittsalter der berufstätigen Ergotherapeuten fiel in den letzten Jahren von 41 auf 39 Jahre. Dagegen stieg es bei den Ergotherapie-Assistenten auf 42 Jahre.

Bezahlung nach Therapieeinheiten $\rightarrow$ Ein weiterer Trend lässt sich bei den Vergütungsmodellen erkennen. Im Vergleich zum Jahr 2000 erhielten 2014 mehr Berufsangehörige anstatt eines festen Gehalts eine Vergütung nach geleisteten Therapieeinheiten. So gaben 44,7\% der befragten Ergotherapeuten an, nach Therapiestunden bezahlt zu werden. Im Jahr 2000 waren es noch 35,1\%. Bei den Ergotherapie-Assistenten hingegen ist eine Bezahlung nach geleisteten Therapieeinheiten der Regelfall [1].

Was die Beschäftigungsform angeht, so ist die Mehrheit des Gesundheitspersonals mit über $80 \%$ angestellt. Die Zahl der Selbstständigen scheint zu sinken.

Ergotherapie hat enormes Wachstumspotenzial $\rightarrow$ Der Berufstrend in den USA zeigt das enorme Wachstumspotenzial der Ergotherapie. Gerade im Hinblick auf zukünftige Herausforderungen, vor denen auch das Gesundheitswesen in Deutschland steht. Dabei sind die Auswirkungen der Ausbildungskosten nicht zu unterschätzen. Denn auch bei uns wird über den steigenden Bedarf an Ergotherapie in der stationären und ambulanten Versor-

\section{5}

\section{Je höher der akademi- sche Abschluss, desto höher das Jahresgehalt.}

gung diskutiert. Um das Leistungsangebot langfristig zu gewährleisten, müssen mehr Ergotherapeuten ausgebildet und angemessen bezahlt werden.

Im Gegensatz zu Deutschland spielt in den USA das Thema Berufsflucht eine untergeordnete Rolle (○ ERGOPRAXIS 3/18, S. 10). Von den Befragten wechselten nur wenige innerhalb der vergangenen zwei Jahre ihre Stelle oder stiegen aufgrund der psychischen und physischen Belastungen ganz aus.

Insgesamt bleibt die Attraktivität der Gesundheitsbranche in den USA hoch. Vor allem wegen der Vergütung und den Social Benefits. Das sind motivierende Zusatzleistungen wie Prämien für die eigene Krankenversicherung oder Altersvorsorge, die angeboten werden, um Fachpersonal zu gewinnen.

Frank Zamath

$\rightarrow$ Literaturverzeichnis

www.thieme-connect.de/products/ergopraxis > „Ausgabe 7-8/18“

\section{舟 Autor}

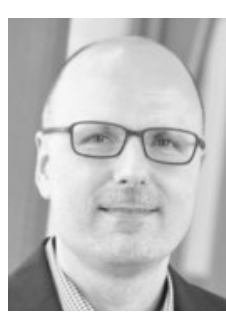

Frank Zamath, Ergotherapeut, arbeitete während seines Studiums an einer High School im US-Bundesstaat Nebraska. Heute ist er an einem Fachkrankenhaus für Psychiatrie, Psychotherapie und Neurologie tätig. Seine Schwerpunkte sind arbeitstherapeutische Versorgung und Leistungsdiagnostik. Seit 2010 ist er im Leitungsteam des DVE-Fachausschusses Arbeit und Rehabilitation. 